\title{
AN ENHANCED METHOD OF LIVER LESION DETECTION USING DEEP NEURAL NETWORK, WATERSHED TRANSFORM AND GAUSSIAN MIXTURE MODEL TECHNIQUES IN MR IMAGES
}

\author{
A.BathshebaParimala ${ }^{1}$, R.S.Shanmugasundaram ${ }^{2}$ \\ ${ }^{1}$ ResearchScholar, Vinayaka Missions Research Foundation, Salem \\ ${ }^{2}$ Professor, Vinayaka Missions Kirupananda Variyar Engineering College, Salem
}

Article DOI: https://doi.org/10.36713/epra7055

DOI No: $10.36713 /$ epra7055

\begin{abstract}
Cancer of the liver is one of the leading causes of death all over the world. Physically recognising the malignancy tissue is a difficult and time-consuming task. In the future, a computer-aided diagnosis (CAD) will be used in dynamic movement to determine the precise position for care. As a result, the primary goal of this research is to use a robotized approach to precisely identify liver cancer. Methods: In this paper, we suggest a new approach called the watershed Gaussian based deep learning (WGDL) strategy for accurately portraying malignant growth sores in liver MRI images. This project used a total of 150 images to build the proposed model. The liver was first isolated using a marker-controlled watershed division scale, and the malignancy-induced injury was then divided using the Gaussian mixture model (GMM) algorithm. Different surface highlights were removed from the sectioned locale after tumour division. These jumbled highlights were fed into a deep neural network (DNN) classifier for a computerised classification of three types of liver cancer: haemangioma (HEM), hepatocellular carcinoma (HCC), and metastatic carcinoma (MET). The following are the outcomes: Using a Deep Neural Network classifier and an unimportant approval deficiency of 0.053 during the characterization period, we were able to achieve a grouping precision of 98.38 percent at 150 ages. The system in our proposed approach is suitable for testing with a large data set and can assist radiologists in detecting liver malignant growth using MR images.

KEYWORDS: computer-aided diagnosis (CAD), watershed Gaussian based deep learning, Gaussian mixture model, hepatocellular carcinoma, metastatic carcinoma, Deep Neural Network classifier
\end{abstract}

\section{INTRODUCTION}

Malignant development of the liver is a common cause of death all over the world. Computed tomography (CT) images may be used to precisely identify the harmful tissue [1]. Computer-aided diagnosis can be used for the arrangement of liver disease in the image processing approach to assist the clinician in complex interaction [2]. Image processing and artificial intelligence approaches have potential in research applications for effective characterization of liver malignant development. There are a few algorithms for detecting liver tumours that include district-based approaches, watershed improvement, and an AI approach. For successful order of liver tumours using GLCM based highlights, a mechanised approach based on CAD structure has been accounted for [3]. Huang et al. [4] proposed a CAD-based diagnosis method for portioning and ordering liver tumours inside nonupgraded CT images. They used auto-covariance surface highlights to group the tumour with an accuracy of 81.7 percent in their augmentation work [5]. Centered on a molecule swarm enhancement process, Ji et al. [6] proposed an important computational model for the clinical diagnosis of hepatocellular carcinoma. To detect liver malignancy with greater precision, a novel and efficient improved approach based on event streamlining (IO) and SVM has been accounted for [7]. In CT images, Li et al. [8] 
used an edge-based distance regularised level-set assessment approach that effectively segmented the pimple, tumour, calculi, and normal liver. In CT images of section liver tumours, a multi-channel fully convolutional network (MC-FCN) model provides better accuracy [9]. In AI approaches, the GLCM and other element extraction procedures are used to obtain observable highlights. [nine] The existing state of division approaches focused on clinical liver images is presented in this paper. Similarly, this paper focuses on various division and arrangement approaches that have been suggested to diagnose a variety of liver diseases. (\#14)

\section{METHODS}

Watershed Gaussian based deep learning (WGDL) procedure is a proposed CAD model that uses force-based division to accurately describe malignant growth injury in CT images of the liver. The liver was separated from other mid-region organs using the watershed division, and the disease tissue was divided using the Gaussian mixture model (GMM). To distinguish HEM, HCC, and MET types of liver tumours, the eliminated observable, textural, and geometrical highlights were arranged using a DNN classifier. 2.1.1.

\section{DATASET}

The imaging centre of IMS and SUM Hospital in India provided a total of $225 \mathrm{CT}$ images of liver malignant development. These were taken from 75 patients (50 males and 25 females) with hemangioma (HEM), 50 patients (25 males and 25 females) with hepatocellular carcinoma (HCC), and
25 patients ( 15 males and 10 females) with metastatic carcinoma (MET). The images were taken on a GE clinical platform CT examine machine with segment thicknesses ranging from 0.5 to $1.5 \mathrm{~mm}$ with the target of. The investigation was carried out on a PC with an Intel $(\mathrm{R})$ center-based processor and $8 \mathrm{~GB}$ RAM, running MATLAB 2015a.

\section{FLOW CHART FOR PROPOSED MODEL}

\subsection{Segmentation}

The affected sections are fragmented using an area-based division process. The 3D-CNN algorithm is used to arrange and characterise Liver 3D-CT and 3D-US images separately. The results are then analysed, and it is discovered that $3 \mathrm{D}-\mathrm{CT}$ is more accurate than 3D-US. TensorFlow and Python's Keras are used to implement the proposed model[15].

\subsection{Watershed transform}

The Watershed transition is a topographybased division algorithm based on the concept of topography $[11,12]$. The grayscale images are interpreted as spatial alleviation in this algorithm, with a neighbouring least regarded as a catchment cup. When water overflows, it creates a boundary and forms a watershed. This process resulted in a complete image division. The image structure is obtained using the morphological operation. The structure clamour and various objects from the grayscale image are often smothered by this operation. For a smooth structure of the limit, we used watershed change on the inclination picture. 


\section{EPRA International Journal of Research and Development (IJRD)

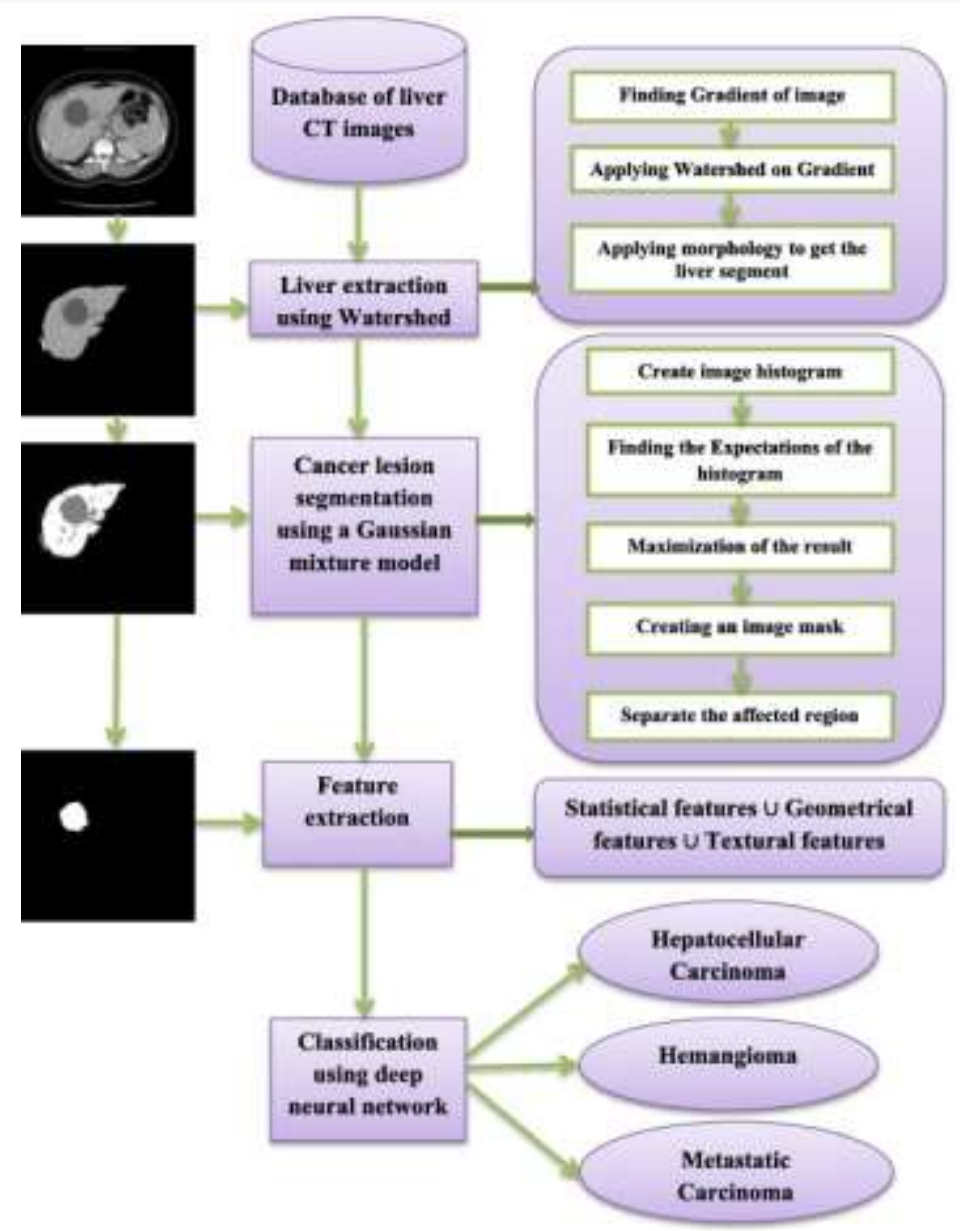

Fig 1 Flow chart

\subsection{Gaussian Mixture Model}

The image is presented in the context of a system in which each part addresses one pixel. The image pixels are treated as a random variable in the Gaussian mixture model (GMM) [13], and are represented by the variable $\mathrm{x}$, which refers to a threedimensional variable with RGB values. A weighted amount of Gaussian dispersion by (5) is used to resolve the image's probability, where $\mathrm{k}$ is the absolute number of districts and the set is the set of loads that satisfy the condition. The term refers to the ith area's Gaussian conveyance with the mean and standard deviation, respectively. (6) To evaluate GMM, we must first determine the model's parameter. The greatest likelihood assessment is the most commonly used method for determining the GMM parameter. The assessment's main goal is to increase the likelihood of the GMM dataset. For evaluation, the expectation boost (EM) algorithm is used. 


\section{EXPERIMENTAL RESULT}
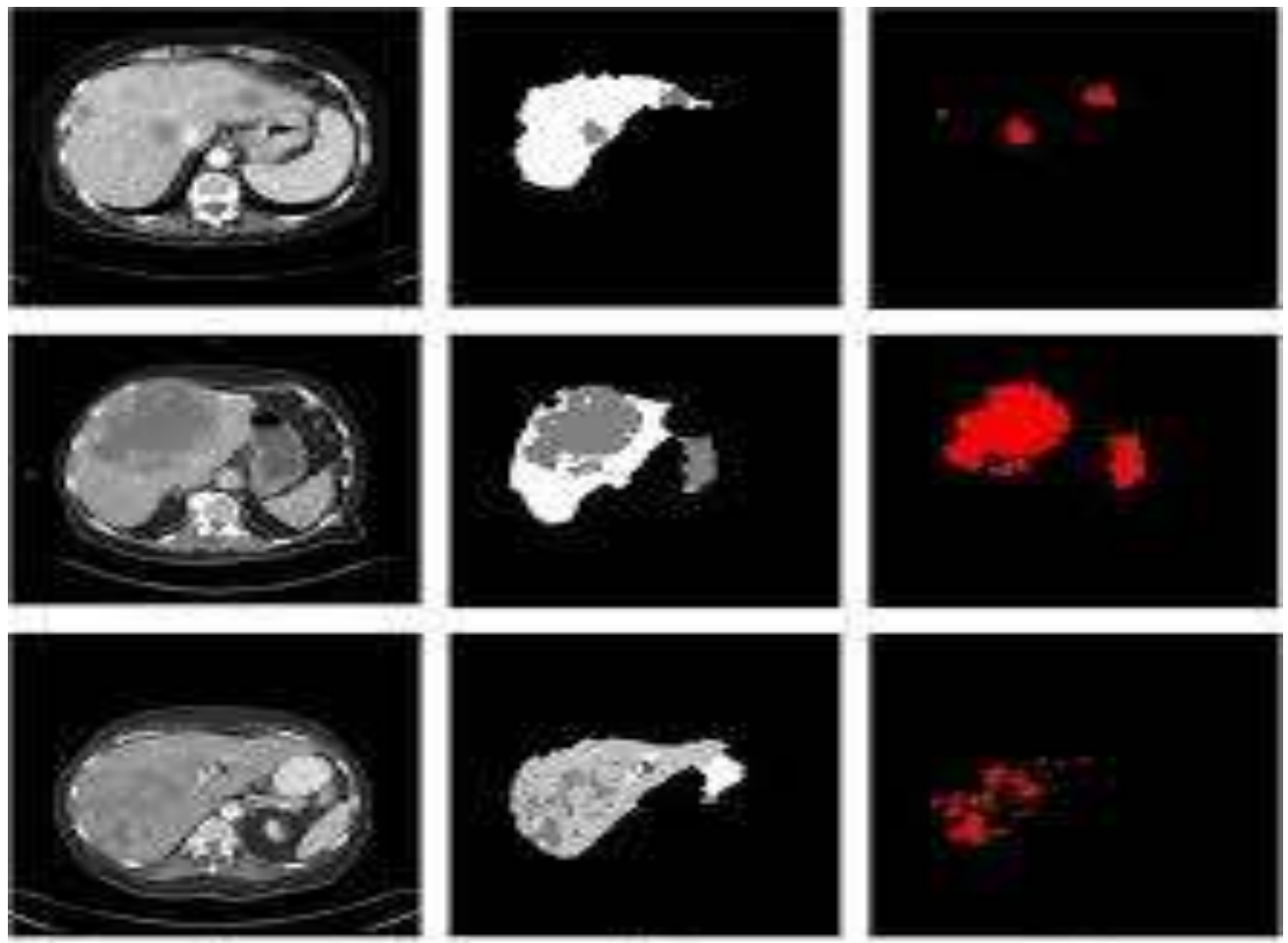

$|a|$

Wh

Fig 2.Deep learning based Liver cancer detection

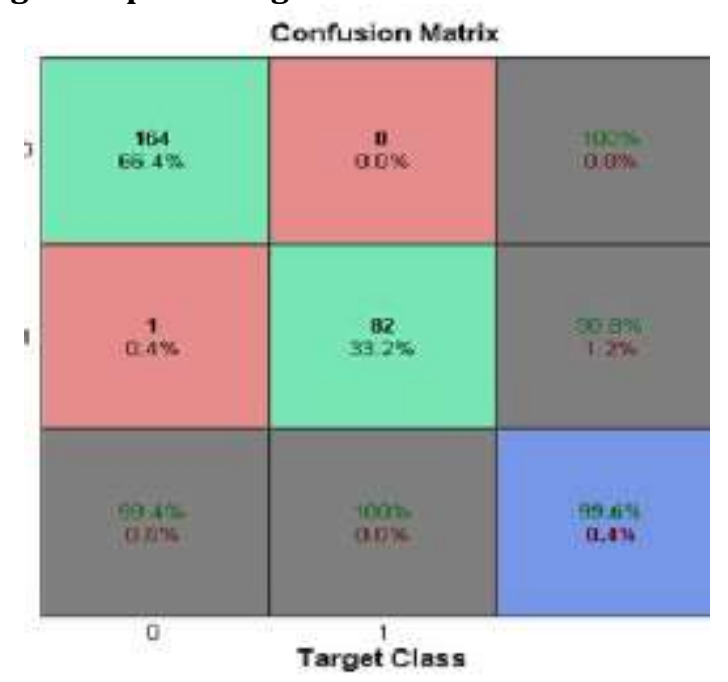

Fig 3 Confusion Matrix 


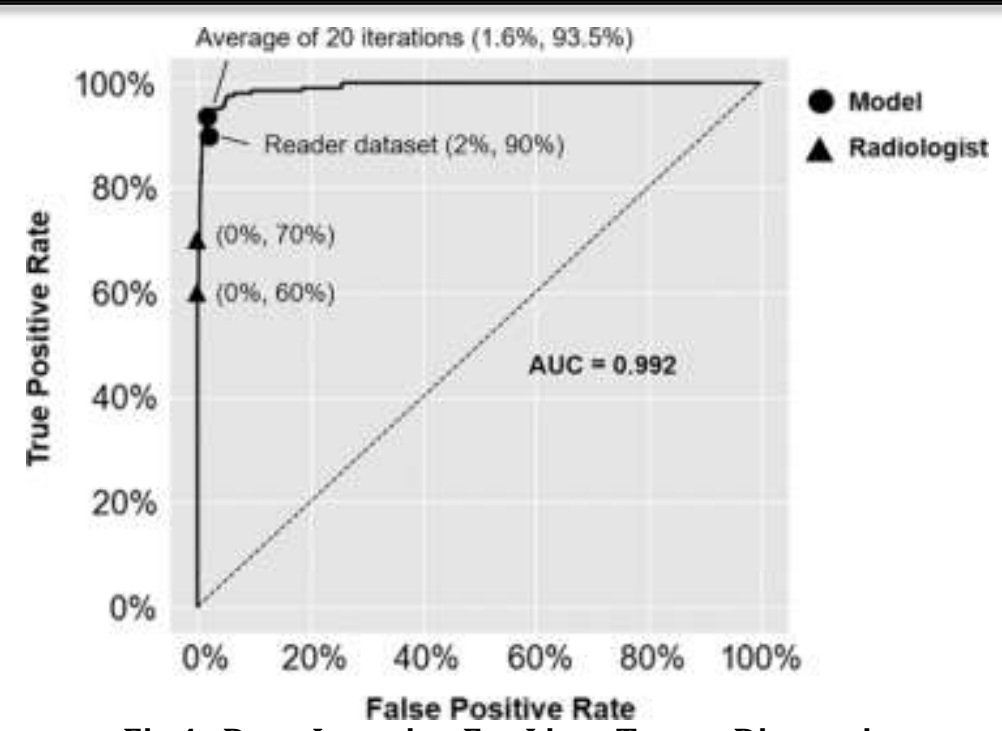

\section{CONCLUSION}

Another technique for the programmed identification of liver tumours in CT images is discussed in this paper. To reliably detect disease injury, this approach uses a marker-controlled watershed shift and a Gaussian mixture model. The proposed algorithm has been validated using a consistent clinical dataset collected from a variety of patients in a clinical setting. The primary advantage of this robotized detection method is that it achieved the highest accuracy of 99.38 percent by using a deep neural network classifier with minor approval misfortune. The first use of the DNN model in a detection measure is in the detection of liver tumours. As a result, the proposed approach is an efficient method for detecting the malignancy locale from CT images of the liver, which will be useful in clinical diagnosis and complex interaction for early diagnosis of indications. The work's fundamental limit is the calculation of the volumetric size of the injury, which can be shaped by creating a 3-D cross section structure from various picture cuts.

\section{REFERENCES}

1. C. Bartolozzi, S. Ciatti, and E. Lucirelli (1981). In the assessment of central liver disease, ultrasound and computer tomography are used. 545-548 in ActaRadiol.

2. I. Kononenk (2001). Artificial intelligence for clinical diagnosis. Best of class and perspective in history. 89-109 in ArtifIntell Med.

3. Chen, E., Chung, P., Chen, C., Tsai, H., and Chang, I. Chen, E., Chung, P., Chen, C., Tsai, H., and Chang, I. (1998). A demonstrative system for CT liver image classification that has been programmed. 45,783-94 in IEEE Trans Biomed Eng.

4. Y. Huang, J. Chen, and W. Shen (2004). In nonenhanced CT images, computer-aided diagnosis of liver tumours. 9, 141-50 in J Med Phys.

5. Huang, Y., Chen, J., and Shen, W. Huang, Y., Chen, J., and Shen, W. (2006). Surface investigation of non-improved computed tomography images to diagnose hepatic tumours. 713-20 in AcadRadiol.

6. Z. Ji and B. Wang (2014). Using a PSO-based various levelled include selection algorithm, distinguishing possible clinical conditions of hepatocellular carcinoma. 1-12 in Biomedical Research International.

7. H. Jiang, R. Zheng, D. Yi, and D. Zhao (2013). Centered on CPSO-SVM and IO, an epic multioccurrence learning method for liver disease recognition on stomach CT images. 1-10 in Comput Math Methods in Medicine.

8. C. Li, C. Xu, C. Gui, and M. Fox (2010). The implementation of distance regularised level sets and their application to image division. 3243-54 in IEEE Transactions on Image Processing.

9. Sun, C., Guo, S., Zhang, H., Li, J., Chen, M., Ma, S., Jin, L., Liu, X., Liu, X., Liu, X., Liu, X., Liu, X., Liu, X., Liu, X., Liu, X., Liu, X., Liu, X., Liu, $X$. (2017). CT images based on FCNs were upgraded by programmed division of liver tumours from multiphase differentiation. 58-66 in ArtifIntell Med.

10. R. Haralick, K. Shanmugam, and I. Dinstein (1927). Image characterization with textural highlights. 3,610-21 in IEEE Trans Syst Man Cybern.

11. Z. Hu, J. Tang, Z. Wang, K. Zhang, L. Zhang, and $Q$. Sun (2018). A research on deep learning for disease detection and diagnosis using images. Example Recognition, vol. 83, no. 134, pp. 134149.

12. H. Masoumi, A. Behrad, M.A. Pourmina, and A. Roosta (2012). An iterative watershed algorithm and artificial neural network were used to programme liver division in MRI images. 429- 
437 in Biomedical Signal Processing and Control.

13. A. Chattaraj, A. Das, and M. Bhattacharya. 13. Chattaraj, A., Das, A., and Bhattacharya, M. (2017). Marker-controlled watershed algorithm divides mammographic images. 1000-1003 at the IEEE International Conference on Bioinformatics and Biomedicine (BIBM).

14. R. S. Shanmugasundaram, A. Bathsheba Parimala Medical Image Processing was used to assess the classification of liver disease. Volume 8 Issue 5 of the International Journal of Recent Technology and Engineering (IJRTE), ISSN: 2277-3878, January 2020

15. AN ENHANCEDLIVER STAGES CLASSIFICATION IN $3 D$-CTAND $3 D$ USIMAGESUSING GLRLM AND 3D-CNNA, by A. Bathsheba Parimala and $R$. $S$. Shanmugasundaram. Volume 12, Issue 2, February2021, pp. 171-184, Article ID: IJEET 1202016. Global Journal of Electrical Engineering and Technology (IJEET) Volume 12, Issue 2, February2021, pp. 171-184, Article ID: IJEET 1202016. 